\title{
Towards a Webpage-based Bibliographic Manager
}

\author{
Dinh-Trung Dang, Yee Fan Tan, and Min-Yen Kan \\ Department of Computer Science, School of Computing, \\ National University of Singapore, Singapore, 117590 \\ \{dangdinh, tanyeefa, kanmy\}@comp.nus.edu.sg
}

\begin{abstract}
We present ForeCiteNote, an application that organizes personal digital collections of research articles. It is architected as a single HTML page with embedded Javascript that runs within a web browser. On top of standard annotation and tagging functionality, it also supports both online and offline usage patterns, including local storage of the paper collection.
\end{abstract}

\section{Introduction}

Scholarly information has greatly expanded in recent years, and while the exact balance of causes responsible for this are difficult to pinpoint, certainly open-access digital libraries and timely dissemination play a part [1]. While such free flow of information presents an unparalleled learning opportunity, it also presents challenges in managing these information sources, especially for beginning researchers.

To focus on the needs of researchers, we performed a focus group study at two academic institutions, involving 12 participants from the French National Institute for Research in Computer Science and Control (INRIA) and 7 from the National University of Singapore (NUS). Each participant completed ten questions on how they performed their own bibliographic management. The findings showed that around $60 \%$ store their papers both on- and off-line and have some difficulty organizing them. $43 \%$ percent reported using offline applications to take notes on research papers. Over $60 \%$ usually access their data when they are away, and do not view their own method as convenient nor systematic. $83 \%$ of those surveyed manually enter all the metadata (e.g., author, date, conference) when annotating papers. A good bibliographic management application (BMA) is thus needed for organizing documents: allowing researchers to collect, retrieve, annotate and organize citations and digital copies of scholarly articles.

\section{Related Work}

BMAs are not a new topic; in fact there are many commercial systems to cover such needs. A comprehensive survey of all solutions is beyond the scope of this paper. We focus on a few current major systems that represent the spectrum available. EndNote (http://www.endnote.com/) is the canonical example of the standalone BMA. It features comprehensive import and export functionality, organization of digital files, and word processing program integration. On the other hand, web-based applications such as CiteULike (http://www.citeulike.org/) [2] and BibSonomy 
(http: //www.bibsonomy.org/) [3] have central servers housing users' bibliographies, thereby promoting sharing and collaborative annotation and tagging among users. Connotea (http://www. connotea.org/) [4] also facilitates offsite web use through browser extensions that can send annotations and references back to the central server. Zotero (http: / / www . zotero.org/) takes this idea further by embedding the whole BMA within a Firefox extension, eschewing a server based solution entirely.

Each architecture has distinct advantages and compromises. A standalone application offers the maximum flexibility but requires the user to run a separate application and is prone to data loss (if the user's hard drive crashes). Server based BMAs make the provider responsible for data integrity and immediately provide collaborative possibilities. However, this architecture cannot manage a user's local digital collection and requires access to the web at all times. Browser extension based BMAs play well both on- and off-line, but increase the memory footprint of the browser and also have the problem of data loss. Also, as the system is tied to a specific web browser instance, their library does not port with them if the user changes browser or computer.

From the above architectural discussions, four central issues emerge: 1) accessibility both on- and off-line, 2) portability across machines, 3) preventing data loss, and 4) local file management. ForeCiteNote aims to solve these issues, by basing its bibliographic management on a different technology: the Javascript-enabled local web page.

\section{ForeCiteNote: A TiddlyWiki-based Bibliographic Manager}

ForeCiteNote is a client-side solution, with a supporting server component, that addresses the above issues. The server component provides data integrity by allowing users to synchronize or backup their annotations and references. It also features online access to the user's data and is capable of adding references and annotations, similar to other server-based BMAss. The key difference is in ForeCiteNote's client-side component, which is a modified version of a TiddlyWiki.

TiddlyWiki (http: / / www . tiddlywiki . com/) is a self-contained HTML page which uses embedded Javascript to give the page the functionality to act as a wiki or a blog. A user can add entries to keep notes or progress logs to the TiddlyWiki in entries known as tiddlers. As it is a single, standard HTML file, it can be uploaded to a web server so that it can be accessed anywhere with an internet connection, and downloaded to other PCs, resolving Issue 1. When used off-line (locally on the user's computer), the permissions afforded by the file: protocol allows new and modified tiddlers to be saved by overwriting the TiddlyWiki itself on the user's disk. Since TiddlyWiki is just a single, small $(\sim 400 \mathrm{~KB})$ web page and requires no installation, users can store their own TiddlyWiki on a local USB drive and carry it around to view on any offline computer equipped with web browser. This makes TiddlyWiki eminently portable and thus resolves Issue 2 . To resolve the remaining two issues, we had to modify the basic TiddlyWiki, resulting in the final ForeCiteNote client, which we describe next.

To solve Issue 3, ForeCiteNote features a synchronization feature that allows the client ForeCiteNote to send its annotations and entries to a ForeCiteNote server. The synchronization engine first allows the upload any entries to the server and then allows 

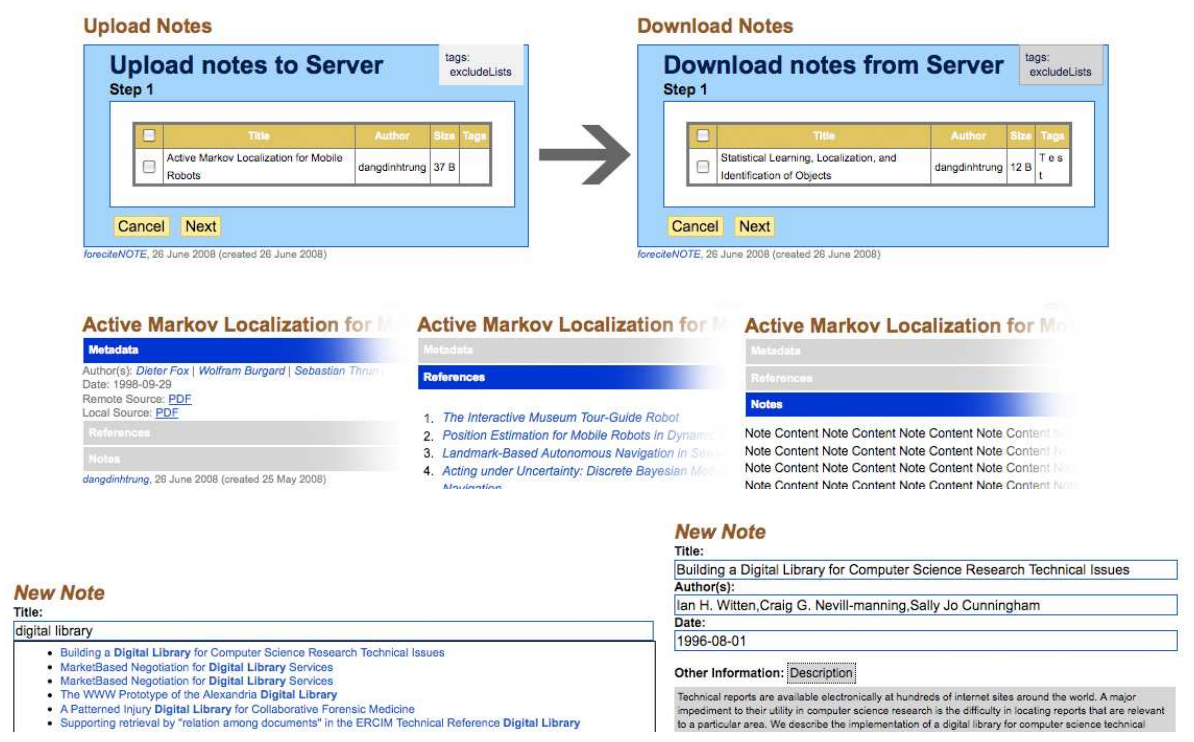

Fig. 1. Top row: Synchronization wizard. Middle row: New display format for tiddlers. Bottom row: Suggestion lists (left) and metadata autocompletion (right).

the user to download any missing entries (Figure 1, top row) illustrates this. In the drastic case where the user has lost their client-side web page, one obtains an empty client and synchronizes it to download all of their entries.

To solve Issue 4, the ForeCiteNote client also manages a directory where it stores local documents (named paperlib). As users may want to browse the local collection outside of the ForeCiteNote webpage application, care was taken to organize local documents in a useful and domain-independent way. According to [5], researchers identified the author, year and title as the three most prominent metadata that identify a scholarly document. ForeCiteNote structures its directory accordingly, in an iTunes-like directory structure: where the author and year are the first and second respective subdirectory level structures within the local document directory, and where the document itself is renamed after the title of the document.

For documents that the ForeCiteNote server knows the metadata and a location of an open access copy, the user can use the client to download and store a copy into the local document directory. The user can similarly ask the ForeCiteNote client to store a copy of other documents that the user has a copy, but which is unknown to the server. Sharing a document collection is as simple as sending the directory tree to a collaborator, which can be done by using archiving utilities and email. Also, since there are many desktop search applications, we intentionally left out local collection search functionality from the client.

We also customized ForeCiteNote to improve upon its usability for recording research notes. First, we modified ForeCiteNote's tiddler format, to better display and 
alternate between displaying a work's metadata or its annotations in one place, as in Figure 1 (middle row).

Second, in our focus groups, metadata entry was identified as a severe bottleneck in annotation. Users clearly wanted references chunked into specific fields (e.g., author, title, year) but did not want to manually enter all of these fields. When the client has internet access, it can query the server to retrieve pertinent metadata to perform metadata autocompletion. When the user first creates a new entry for a paper, they start by typing in the document title. After three letters have been typed in, the client queries the server for any autocompletion matches based on papers known to the system. These are shown to the user, as in Figure 1 (bottom row, left). The user can then either ignore the suggested matching list or pick the document's title if shown. If a suggested known paper is chosen, the client retrieves the metadata from the server, including the abstract and any open-access location, and fills in the appropriate fields in the entry, as in Figure 1 (bottom row, right).

\section{Conclusion}

We have introduced ForeCiteNote, a webpage-based bibliographic manager. As an adaption of the revolutionary TiddlyWiki software, ForeCiteNote not only allows researchers to collect, annotate and organize citations and digital copies of scholarly articles and also a means to retrieve them, but also guarantees the accessibility both online and offline and portability across machines.

ForeCiteNote has undergone several rounds of informal user design testing and has incrementally been improved to its current state, reported here. Current work focuses on a long-term, longitudinal assessment of ForeCiteNote's usability. We expect to release the application for beta testing soon. Future work includes extending the ForeCiteNote server functionality to support the collaborative work of distributed groups.

\section{References}

1. Prosser, D.C.: Scholarly communication in the 21 st century - the impact of new technologies and models. J. Serials Community 16 (2003) 163-167

2. Emamy, K., Cameron, R.: CiteULike: A researcher's social bookmarking service. Ariadne (2007)

3. Hotho, A., Jschke, R., Schmitz, C., Stumme, G.: Bibsonomy: A social bookmark and publication sharing system. In: Conceptual Structures Tool Interoperability Workshop at 14th ICCS Conf. (2006) 87-102

4. Anonymous: Join a social revolution. Nature 436 (2005) 1066

5. Day, M.Y., Tsai, T.H., Sung, C.L., Lee, C.W., Wu, S.H., Ong, C.S., Hsu, W.L.: A knowledgebased approach to citation extraction. In: IRI. (2005) 50-55 